\title{
A Dual Staining Method for Neutral Carbohydrates and DNA (Deoxyribonucleic Acid) Using Periodic Acid-Thiocarbohydrazide- Silver Protein-Physical Development (PA-TCH-SP-PD) and Feulgen (Hot HCl-Schiff) Techniques
}

\author{
By \\ Takashi UEDA, Satoshi TERADA, Osamu FUJIMORI and Kazuyori YAMADA \\ 2nd Department of Anatomy, Nagoya City University Medical School, 1 Kawasumi; Mizuho-cho, Mizuho-Ku, \\ Nagoya 467, Japan \\ - Received for Publication, August 24, $1992-$

\begin{abstract}
Key Words: Dual staining method, Periodic acid-thiocarbohydrazide-silver protein-physical development, Feulgen reaction, Neutral carbohydrates, DNA

Summary: A dual staining method was established for the histochemical detection of neutral carbohydrates and deoxyribonucleic acid (DNA) in light microscopy. The method consisted of combined periodic acid-thiocarbohydrazide-silver protein-physical development (PA-TCH-SP-PD) and hot hydrochloric acid (H-HCl)-Schiff (Feulgen) procedures, which produced reaction products of blackish and magenta shades respectively. The present dual staining method is believed to be unusually useful for the light microscopic histochemical studies on neutral carbohydrate-containing cells, since it exhibits a high contrast between these two shades and reveals distinctly the localization of cytoplasmic neutral carbohydrates and nuclear DNA in one and the same tissue section.
\end{abstract}

For the histochemical detection of neutral carbohydrates in light microscopy, a series of reliable methods have been devised. Among them were periodic acid-methenamine silver (PA-MeS) (Gomori, 1946), periodic acid-Schiff (PAS) (McManus, 1948; Lillie and Fullmer, 1976, Pearse, 1985), periodic acid-phenylhydrazone-formazan (PA-PH-FZ) (Seligman et al., 1949; Stoward and Mester, 1964; Stoward, 1967) and periodic acid-m-aminophenol-Fast Black salt K (PA-AP-FBK) (Lillie et al., 1961; Lillie, 1962; Yamada, 1978). Of these techniques, the PAS method has been found to be the most reliable and has hence been used widely for the light microscopic histochemistry of neutral carbohydrates. In the PAS method, however, the sensitivity of Schiff's reagent for PA-engendered dialdehydes has been recognized to be limited (Stoward and Mester, 1964) and the images obtained by the magenta reaction products are relatively vague in contour. In light of such limitations, Nakamura et al. (1985) have established a periodic acid-thiocarbohydrazidesilver protein-physical development (PA-TCH-SPPD) method as a sensitive, precise and reliable technique of choice for the light microscopy of neutral carbohydrates. These authors have, however, not employed any counter-stainings for cell nuclei in combination with the PA-TCH-SP-PD staining method. Thus, the staining method for neutral carbohydrates has been found to be inconvenient for the orientation of individual cells.

To overcome such an inconvenience, in the present study, an attempt has successfully been made to combine the PA-TCH-SP-PD method with hot hydrochloric acid-Schiff (Feulgen) technique and to establish a dual staining procedure for the simultaneous demonstration of neutral carbohydrates and DNA of individual cells in one and the same tissue section. The present dual staining method shows a high contrast between blackish and magenta shades and is regarded as a precise method of choice for the efficient histochemical visualization of cytoplasmic neutral carbohydrates and nuclear DNA in light microscopy.

\section{Materials and Methods}

Preparation of tissues:

A series of organs of the digestive, respiratory and urinary systems were removed from adult SpragueDawley rats of both sexes, which were sacrificed by exsanguination following nembutal anesthesia. These organs included the stomach, duodenum, colon, submandibular and sublingual glands, trachea. 
lung and kidney. Tissue pieces of these organs were fixed in Carnoy's solution at $4^{\circ} \mathrm{C}$ for $4-6 \mathrm{hrs}$, dehydrated in $100 \%$ ethanol, cleared in xylene and embedded in paraffin wax. Sections were cut at a thickness of 4 to $6 \mu \mathrm{m}$ and mounted on glass slides without any adhesives. The tissue sections were then dewaxed in xylene, hydrated in an ethanol series of descending concentrations and subjected to the experimental and control staining procedures detailed below.

\section{Histochemical reagents:}

Of a number of the histochemical reagents used, periodic acid, glacial acetic acid, hydroquinone, citric acid, gum arabic, hydrochloric acid and sodium pyrosulfite were obtained from Katayama Chemical Company (Osaka, Japan), whereas silver protein, silver nitrate and basic fuchsin were purchased from Merck GmbH (Darmstadt, Germany). In addition, thiocarbohydrazide employed was a product of Eastman Kodak Company (New York, U.S.A).

Staining procedures:

I. Experimental staining procedure (PA-TCH-SPPD-H-HCl-Schiff)

Hydrated sections were

1. Immersed in $10 \%$ aqueous periodic acid (PA) for $10 \mathrm{~min}$. at $20^{\circ} \mathrm{C}$;

2. Rinsed in three changes of distilled water;

3. Incubated in $1 \%$ thiocarbohydrazide $(\mathrm{TCH})$ in $10 \%$ acetic acid for $40 \mathrm{~min}$ at $40^{\circ} \mathrm{C}$;

4. Washed in running tap water for $30 \mathrm{~min}$;

5. Rinsed in three changes of distilled water;

6. Immersed in $0.5 \%$ aqueous solution of silver protein (SP) for $30 \mathrm{~min}$ at $20^{\circ} \mathrm{C}$ in a dark box;

7. Washed in running tap water for $30 \mathrm{~min}$;

8. Rinsed in three changes of distilled water;

9. Physically developed (PD) with a solution of the following composition * (Nakamura et al., 1985) for $10-20 \mathrm{~min}$ at $18^{\circ} \mathrm{C}$ in a dark box;

\section{* Solution A:}

$20 \%(\mathrm{~W} / \mathrm{V})$ gum arabic in water $45 \mathrm{ml}$

$10 \%$ silver nitrate aqueous solution $1 \mathrm{ml}$

(The gum arabic solution was centrifuged at $19,000 \mathrm{rpm}$ for $30 \mathrm{~min}$ at $0^{\circ} \mathrm{C}$ and supernatant used.)

Solution B: distilled water $15 \mathrm{ml}$

$\begin{array}{ll}\text { hydroquinone } & 200 \mathrm{mg} \\ \text { citric acid } & 300 \mathrm{mg}\end{array}$

The working developing solution was prepared by mixing solutions $\mathrm{A}$ and $\mathrm{B}$ in a dark box immediately prior to use.

10. Rinsed in a photographic fixer (Super Fuji Fix) diluted 1:4 for $5 \mathrm{~min}$;

11. Washed in running tap water for $20 \mathrm{~min}$;

12. Rinsed in distilled water;

13. Incubated in $\mathbf{1} \mathbf{N}$ hydrochloric acid $(\mathrm{HCl})$ for
$10 \mathrm{~min}$ at $60^{\circ} \mathrm{C}$;

14. Rinsed in distilled water;

15. Immersed in Schiff's reagent for $45 \mathrm{~min}$;

16. Rinsed in three changes of freshly prepared bisulfite solution $(0.5 \%$ aqueous solution of sodium pyrosulfite);

17. Washed in running tap water for $5 \mathrm{~min}$;

18. Rapidly dehydrated in an ethanol series of ascending concentrations;

19. Promptly cleared in three changes of xylene; and finally

20. Mounted in neutral Canada balsam.

II. A reverse sequence of the experimental staining procedure (H-HCl-Schiff-PA-TCH-SP-PD)

Hydrated sections were first subjected to the steps 13 to 17 and then to the steps $1-12$ in the experimental staining procedure detailed above.

\section{Control staining procedures}

The following ten procedures were performed as controls in which various steps were omitted.

1. PA-TCH-SP-PD

2. $\mathrm{H}-\mathrm{HCl}-\mathrm{Sch}$ iff

3. PA-TCH-SP-H-HCl-Schiff

4. PA-TCH-PD-H-HCl-Schiff

5. PA-SP-PD-H-HCl-Schiff

6. TCH-SP-PD-H-HCl-Schiff

7. SP-PD-H-HCl-Schiff

8. PD-H-HCl-Schiff

9. PA-TCH-SP-PD-H-HCl

10. PA-TCH-SP-PD-Schiff

\section{Results}

In the present experimental staining procedure (PA-TCH-SP-PD-H-HCl-Schiff), intra- and extracellular neutral carbohydrates and nuclear DNA were colored in black or brown and magenta shades respectively. Thus, black or brown reaction products were visualized on neutral carbohydrates involved in both the intra- and extracellular structures of the rat stomach (Fig. 1), duodenum, colon (Fig. 2), submandibular and sublingual glands, trachea, lung (Fig. 3) and kidney (Fig. 4). In nuclear DNA of all the cells contained in the tissues of these organs, magenta reaction products were obtained following the experimental dual staining procedure (Figs. 1-4).

If the tissues of all the organs examined were subjected to the reverse sequence of the dual staining (H-HCl-Schiff-PA-TCH-SP-PD), nuclear DNA of all the cells involved was stained in significantly paler magenta shades than those obtained following the experimental dual staining procedure (PA-TCH$\mathrm{SP}-\mathrm{PD}-\mathrm{H}-\mathrm{HCl}-\mathrm{Schiff})$. In the tissues subjected to 
the reverse sequence of the dual staining, however, the intra- and extracellular neutral carbohydrates revealed black or brown shades, which were similar in intensity to those obtained following the experimental dual staining procedure (PA-TCH-SP-PD$\mathrm{H}-\mathrm{HCl}$-Schiff).

When the tissues of all the organs examined were stained with the control procedure 1 (PATCH-SP-PD), only the intra- and extracellular neutral carbohydrates were colored in black or brown shades (Fig. 5), whereas only the nuclear DNA involved was reacted in magenta shades following the control procedure 2 ( $\mathrm{H}-\mathrm{HCl}-\mathrm{Schiff})$ (Fig. 6). If the tissues of all the organs examined were subjected to PA-TCH-SP-H-HCl-Schiff, the intra- and extracellular neutral carbohydrates were colored in brown or yellowish brown shades, even though the nuclear DNA involved was stained in magenta shades.

In the tissues of all the organs examined, the control procedures 4 (PA-TCH-PD-H-HCl-Schiff), 6 (TCH-SP-PD-H-HCl-Schiff), 7 (SP-PD-H-HClSchiff) and 8 (PD-H-HCl-Schiff) failed to color any intra- and extracellular neutral carbohydrates involved, whereas the nuclear DNA contained was stained in magenta shades. In the tissues of all the organs examined subjected to the control procedure 5 (PA-SP-PD-H-HCl-Schiff), both the intra- and extracellular neutral carbohydrates and nuclear DNA were colored in magenta shades.

In the tissues treated with the control procedure 9 (PA-TCH-SP-PD-H-HCl) or 10 (PA-TCH-SP-PDSchiff), the nuclear DNA involved failed to stain at all, even though the intra- and extracellular neutral carbohydrates were ordinarily colored in black or brown shades.

\section{Discussion}

In a previous study on the PA-TCH-SP-PD staining method, Nakamura et al. (1985) has substantiated that it is an unusually sensitive and specific technique for the histochemical demonstration of vicinal diols of neutral carbohydrates in light microscopy.

It has been well established that $\mathrm{H}-\mathrm{HCl}-\mathrm{Schiff}$ (Feulgen) staining technique is a highly specific method for the histochemical detection of DNA in light microscopy (Feulgen and Rosenbeck, 1924; Bancroft, 1967; Pearse, 1985; Yamada, 1987).

As the results obtained in the present study reveal, the experimental dual staining method (PA-TCHSP-PD-H-HCl-Schiff) has been found to color the intra- and extracellular neutral carbohydrates and nuclear DNA in black or brown and magenta shades respectively, which exhibit a high contrast between the both. The combination of the two staining methods in this sequence did not yield any unfavorable effects upon the respective reaction products with each other. Thus, the present dual staining method (PATCH-SP-PD-H-HCl-Schiff) is regarded as being a precise and reliable light microscopic technique for the histochemical detection of vicinal diol-containing neutral carbohydrates and nuclear DNA.

The reverse sequence of the dual staining method (H-HCl-Schiff-PA-TCH-SP-PD) appears to be inappropriate, since in the reverse sequence the magenta shades of the nuclear DNA was significantly pale, due perhaps to the suppressive effects of the reagents used for ensuing PA-TCH-SP-PD staining technique upon the magenta colored reaction products of the foregoing $\mathrm{H}-\mathrm{HCl}$-Schiff method.

The results obtained by the majority of the control procedures for the present dual staining method (PA-TCH-SP-PD-H-HCl-Schiff) could be taken to substantiate its superior properties such as the precision, specificity, reliability and reproducibility.

Taken altogether, the present dual staining method (PA-TCH-SP-PD-H-HCl-Schiff) can be postulated to be an excellent technique of choice for the simultaneous histochemical demonstration of neutral carbohydrates and nuclear DNA in light microscopy.

\section{References}

1) Bancroft, J. D.: Nucleic acid. In An Introduction to Histochemical Technique, pp. 136-147, Butterworths, London, 1967.

2) Feulgen, R. and Rosenbeck, H.: Mikroskopisch-chemischer Nachweis einer Nukleinsäure von Typus der Thymonucleinsäure und darauf beruhende elektive Färburg von Zellkernen in Mikroskopischen Präparaten. Z. Physiol. Chem., 135: 203-248, 1924.

3) Gomori, G.: A new histochemical test for glycogen and mucin. Amer. J. Clin. Path., 16: 177-179, 1946.

4) Lillie, R. D., Glimer, P. R. Jr. and Welsh, A. A.: Black periodic and black Bauer methods for tissue polysaccharides. Stain Technol., 36: 361-363, 1961.

5) Lillie, R. D.: The histochemical reaction of arylamines with tissue aldehydes produced by periodic and chromic acids. J. Histochem. Cytochem., 10: 303-314, 1962.

6) Lillie, R. D. and Fullmer, H. M.: Polysaccharides; mucins. In Histopathologic Technic and Practical Histochemistry. 4th edn, pp. 611-678, McGraw-Hill, New York etc., 1976.

7) McManus, J. F. A.: Histological and histochemical uses of periodic acid. Stain Technol., 23: 99-108, 1948.

8) Nakamura, M., Kitamura, H. and Yamada. K.: A sensitive method for the histochemical demonstration of vicinal diols of carbohydrates. Histochem. J., 17: 477-485, 1985.

9) Pearse, A. G. E.: Carbohydrates and Mucosubstances. In Histochemistry, Theoretical and Applied, vol. 2、 4th edn. pp. 675-753, Churchill Livingstone. Edinburgh, London. Melbournc and New York, 1985.

10) Seligman, A. M., Gofstein, R. and Rutenberg, A. M.: Preparation of a radioactive iodotetrazolium salt and its distribution in mice. Cancer Res., 9: 366-369. 1949. 
11) Stoward, P. J. and Mester, L.: Formazan reaction as confirmatory test for aldehyde groups in histochemistry. Nature (Lond.), 31: 488-489, 1964.

12) Stoward, P. J.: Histochemical studies of the formazan reaction. Part I: a theoretical review of some of the factors that can affect the conversion of periodate-reactive mucosubstances into formazans. J. Roy. Micr. Soc., 87: 77-87, 1967.
13) Yamada, K.: Concanavalin A-peroxidase-diaminobenzidineperiodic acid-m-aminophenol-Fast Black salt $\mathrm{K}$ : a method for the dual staining of neutral complex carbohydrates. Histochem. J., 10: 573-584, 1978.

14) Yamada, K.: Detection of nucleic acids. In Histochemistry, Fundamentals and Practices for Beginners, pp. 263-279, Nankodo, Tokyo, 1987. (in Japanese)

\section{Explanation of Figures}

\section{Plate I}

Fig. 1. Parts of the gastric mucous membrane in a rat. Stained with PA-TCH-SP-PD-H-HCl-Schiff. $\times 175$.

Fig. 2. Parts of the colonic mucous membrane in a rat. Stained with PA-TCH-SP-PD-H-HCl-Schiff. $\times 175$.

Fig. 3. Parts of the lung in a rat. Stained with PA-TCH-SP-PD-H-HCl-Schiff. $\times 175$.

Fig. 4. Parts of the renal cortex in a rat. Stained with PA-TCH-SP-PD-H-HCl-Schiff. $\times 175$.

Fig. 5. Parts of the gastric mucous membrane in a rat. Stained singly with PA-TCH-SP-PD. $\times 175$.

Fig. 6. Parts of the gastric mucous membrane in a rat. Stained singly with $\mathrm{H}-\mathrm{HCl}-\mathrm{Schiff} . \times 175$. 


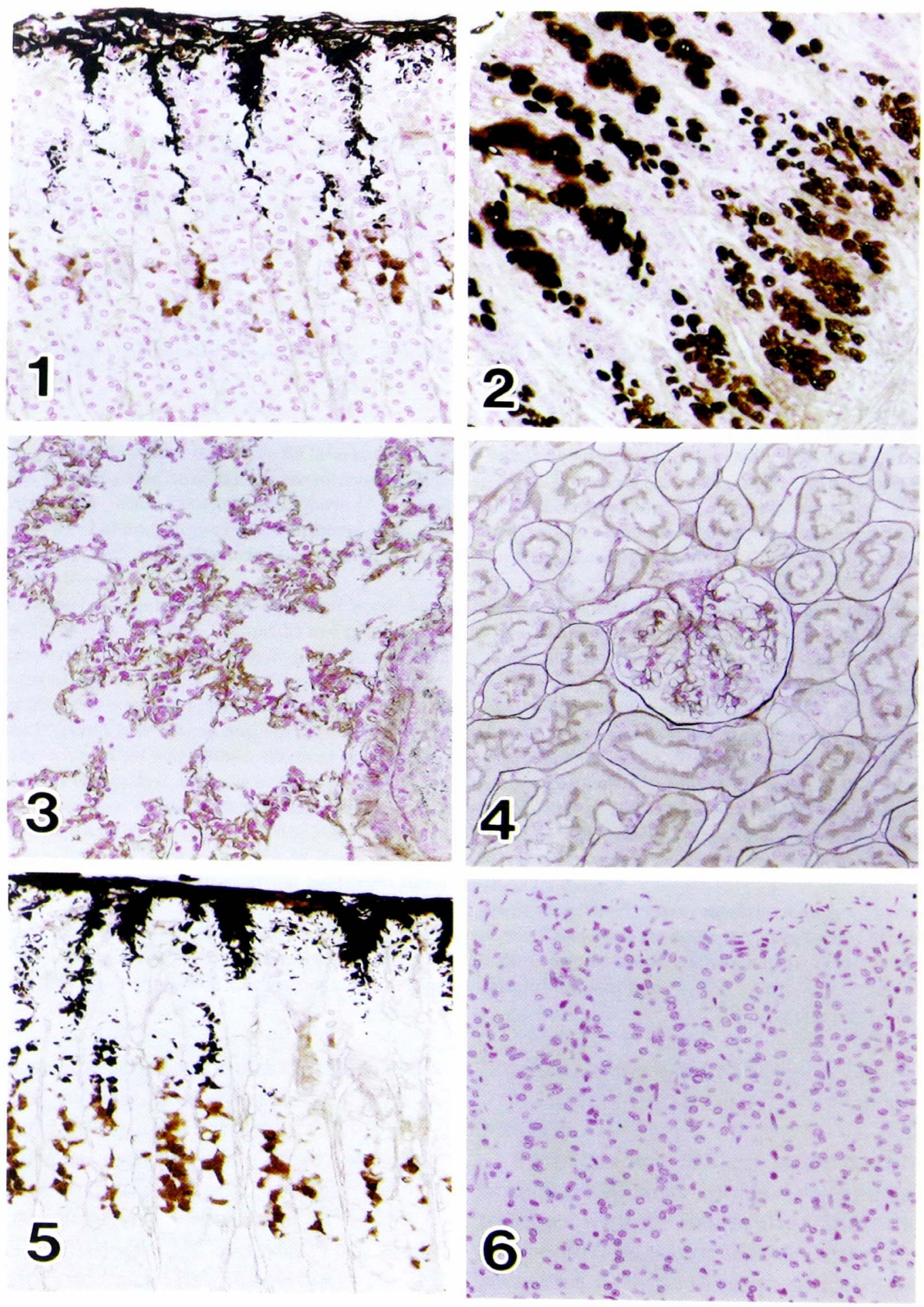

